$\xi_{p}$

\title{
Electromagnetic Shielding Analysis (EMS) of Hybrid Multilayered Woven Fabric at Different Transmitter Distance
}

\author{
N. K. Omar ${ }^{1}$, M.F. Yahya ${ }^{1 *}$, M.R. Ahmad ${ }^{1}$ and M.T. Ali $^{2}$ \\ ${ }^{1}$ Textile Research Group, Faculty of Applied Sciences, Universiti Teknologi MARA, 40450 Shah Alam, Selangor, Malaysia \\ ${ }^{2}$ Antenna Research Group, Faculty of Electrical Engineering, Universiti Teknologi MARA, 40450 Shah Alam, Selangor, Malaysia \\ *Corresponding author E-mail: mfy@salam.uitm.edu.my
}

\begin{abstract}
This paper mainly discusses on the effectiveness of multiple plain-woven fabric towards electromagnetic shielding (EMS). The measurement includes the effect of fabric distance from the transmitter antennas in the shielded enclosure test. The conductive fabric consists of copper cover hybrid yarns produced using hollow spindle spinning machine. The electromagnetic shielding test was performed according to IEEE-299 specification at 2000MHz. The range was selected to reflect mobile phones, wireless fidelity, Bluetooth and GPS transmission range frequencies. Four samples of conductive fabrics were used in the work, as $0^{\circ} / 0^{\circ}, 90^{\circ} / 90^{\circ}, 0^{\circ} / 90^{\circ}$ and $90^{\circ} / 0$, respectively. The fabric sample with $0^{\circ} / 90^{\circ}$ alignment was found to give the best electromagnetic shielding at $-65.79 \mathrm{dBM}$. The initial results also show that the hybrid conductive fabrics have large potential to be utilized for shielding electromagnetic radiation (EMR).
\end{abstract}

Keywords: angle arrangement, conductive fabric, copper cover hybrid yarn, electromagnetic shielding fabrics, multiple-layer

\section{Introduction}

Nowadays, electronic devices have been a part of our daily life. Most of the electronic devices such as mobile phone or medical devices emits the frequency from $200 \mathrm{MHz}$ to $5000 \mathrm{MHz}$ [1]. However, due to the lack of awareness, most people are not concerned about the transmission of electromagnetic radiation. There are 4 mechanisms of electromagnetic waves, (i) reflection, (ii) multiple reflection, (iii) transmission and (iv) absorption [2]. Some of the waves reflect, penetrate and absorb by the shielding material as shown in Figure 1.

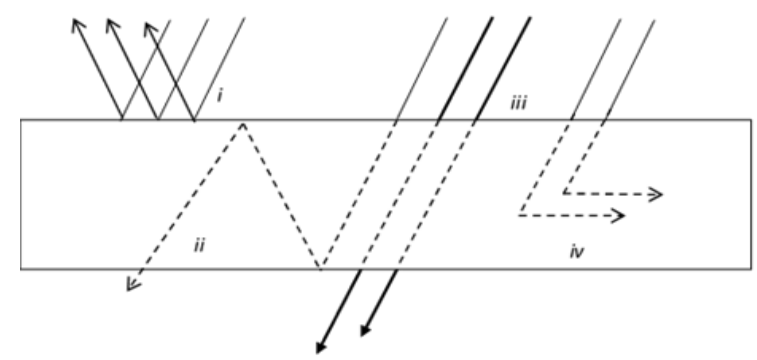

Fig 1: The shielding mechanism

The reflection of electromagnetic waves occurs when the shielding material is made of high conductive material [2]. Multiple reflection of electromagnetic waves occurs due to various surface or phase that are present inside the shield material, for example, foamed material and composites with filler that has large internal surface [2]. The transmission of electromagnetic waves that passes through the shielding without any reduction, and the high trans- mission of electromagnetic waves were observed [2]. The absorption of electromagnetic waves takes place when the shield contains electric or/and magnetic dipoles to interact with electromagnetic field [2].

Electromagnetic radiation gives bad health effects to the human body especially children. Children, especially toddlers are vulnerable to the radiation rays. Most of the parents exposed their children to different frequencies of radiation on a daily basis. Lead contain toxic heavy metals. The high lead level can decrease vitamin D, hemoglobin and possibly causes death [3]. Brain tissues of young children can absorb higher lead than adults because their brain tissue are thinner compare to adult [4-6]. From the previous study, children are more easily to have an increase in blood lead level cause by overlong exposure to electromagnetic radiation [6, 7]. In another study, it was reported that some of the computer users complained of hearing loss, headaches, dizziness, tension, and anxiety symptoms $[8,9]$. These show that exposure to the electromagnetic radiation gives bad health effect to the human body.

In order to overcome the problem, several research works related to the potential of textile structure as an electromagnetic shielding (EMS) has been explored [10-12]. The electromagnetic shielding fabric can be categorized as conductive textile. Conductive textile has more physical advantages compared to metal plate such as more elasticity, light weight, good air permeability and wearable. Moreover, conductive textile as electromagnetic shielding has earned attention among textile researchers as the solution to the issue of electromagnetic radiation $[13,14]$. Examples of textile products with electromagnetic shielding properties are conductive fabrics reinforced thermoplastic composite, copper/PET composite yarn fabric, non-woven microwave absorbing sheet or silver mesh fabric $[15,16]$. In addition, some of the researcher found that by layering the fabrics, the effectiveness of electromagnetic shielding 
can be increase. Perumalraj and Dasaradan [17], found that threeply cotton-copper yarn with twill structure can shield almost $74 \mathrm{~dB}$ with the frequency of $800-2000 \mathrm{Mhz}$. Other than that, Cheng [18] discovered 4 layers with different weave angle of 3/1 twill from copper filament as core wrap with cotton can shield $69.04 \mathrm{~dB}$ with $1800 \mathrm{MHz}$ radiation frequency.

A conductive fabric consists of different types of metal filament. Ortlek [19] found that conductive fabric that consists of metal filament can protect houses and buildings from the emission of electromagnetic radiation originating from cellular phones, televisions, and wireless networks. Conductive fabric helps people from direct contact or exposed to electromagnetic radiation. Some of the conductive fabrics are made by stainless steel, copper, and silver [1, 14, 20-23]. Therefore, researchers produced hybrid yarns, combination of metal filament and textile material. Das [20] found that copper has good properties for electromagnetic shielding (EMS) compared to brass, cellulose, or polyethylene terephthalate (PET). Copper can be used as a shield from electromagnetic radiation (EMR). The study found that $0.09 \mathrm{~mm}$ copper filament showed better shielding performance than $0.1 \mathrm{~mm}$ copper filament [2]. A small-diameter copper filament shows good shielding performance. Copper filament must be combined with another component due to the limitation of physical properties. Copper is rigid and resists to bending when it is used in fabrics [17].

It is important to note that wearability is always an issue with $100 \%$ copper filament yarn. Copper is stiff and thus creates irritation to human skin during wear. To solve the problem, researchers worked on a hybrid yarn that is composed of textile fibre and copper filament [24]. The yarn is made up of two or more types of fibre yarn. Most of the hybrid yarn are suitable to be applied in the technical textile application in the form of smart textile. Some of the researchers used cotton, polyester, and nylon and combined with a metal filament to produce hybrid yarns with shielding effect $[1,14,20-23]$. This project used high tenacity polyester combined with copper filament. High-tenacity polyester yarn can be combined with copper filament as an electromagnetic shielding material for smart textile application. High-tenacity polyester has high durability, toughness, and chemical resistance to hold on the extreme environment, which makes it suitable for use in highperformance application [25].

The following section of the paper discusses the effect of multiangle layered electromagnetic shielding fabric with different distance from the transmitter signal.

\section{Experimental}

\subsection{Material}

The conductive yarn consists of $0.08 \mathrm{~mm}$ diameter cooper filament (36.37 tex) combined with high tenacity polyester yarn (53 tex). The conductive yarn was produced using hollow spindle ring spinning machine as shown in Figure 2 with the optimum speed of 1600rpm. The conductive yarn consists of two components, core and cover as shown in Figure 3. Copper filament will cover up around the high tenacity polyester yarn. This type of yarn is also known as spiral yarn structure. The copper filament was obtained from the open market manufactured by FE Magnet Wire Company, Malaysia, whereas high-tenacity polyester was purchased from a local manufacturer.

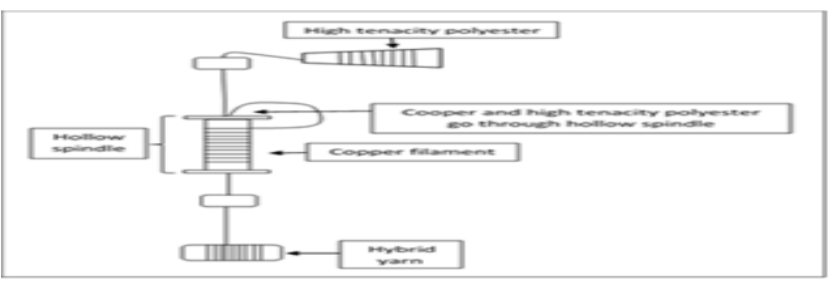

Fig. 2: Process of making conductive yarn

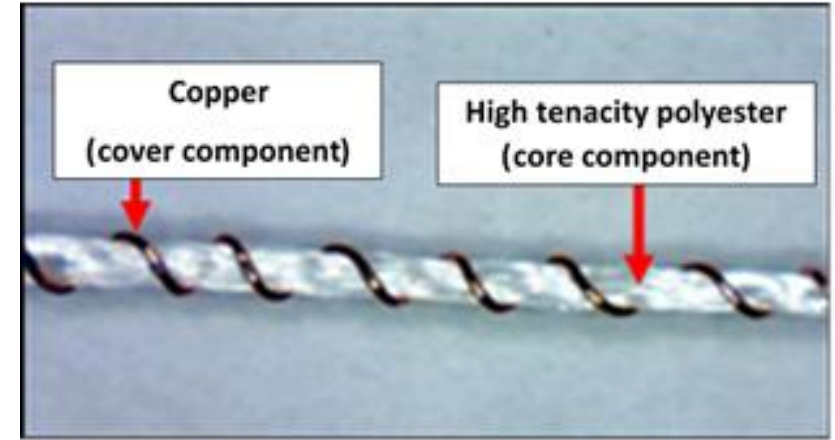

Fig. 3: The spiral structure of conductive yarn consists of copper filament and high tenacity polyester yarn

Once yarn production is completed, the conductive fabric was prepared using conductive yarn (114 tex) as the weft and polyester yarn (150 tex) as the warp, as shown in Figure 4. The density of the fabric is 32 ends per $\mathrm{cm}^{-1}$ and 16 picks per $\mathrm{cm}^{-1}$. The conductive fabrics were produced using DONIER PTS $8 / \mathrm{JC}$ jacquardrapier weaving machine. The main structure of the sample is plain weave structure.

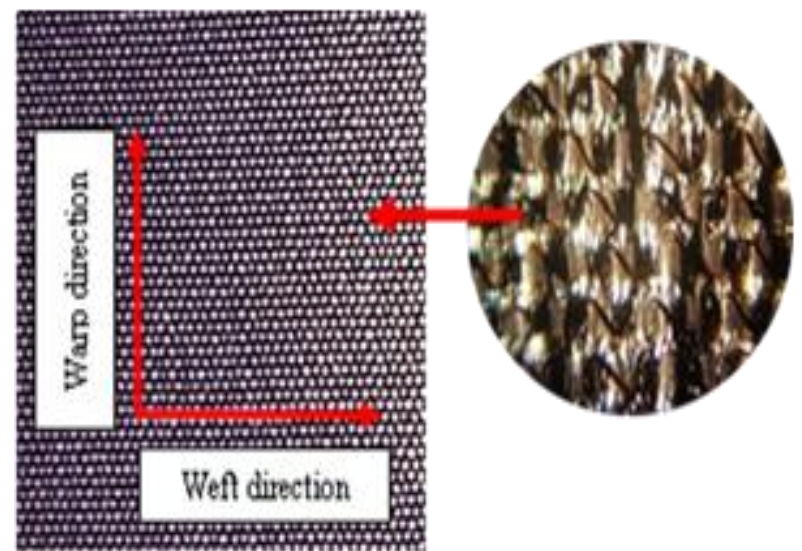

Fig. 4: Conductive fabric of plain weave structure

Table 1: Fabric classification

\begin{tabular}{|c|c|c|c|c|c|c|c|c|}
\hline $\begin{array}{c}\text { Fabric } \\
\text { Id }\end{array}$ & $\begin{array}{c}\text { War } \\
\text { p } \\
\text { yarn }\end{array}$ & $\begin{array}{l}\text { Weft } \\
\text { yarn }\end{array}$ & $\begin{array}{c}\text { Wa } \\
\text { rp } \\
\text { tex }\end{array}$ & $\begin{array}{c}\text { We } \\
\text { ft } \\
\text { tex }\end{array}$ & $\begin{array}{c}\text { End } \\
\text { s/ } \\
\text { cm }\end{array}$ & $\begin{array}{c}\text { Pick } \\
\text { s/ } \\
\text { cm }\end{array}$ & $\begin{array}{l}1^{\text {st }} \\
\text { lay } \\
\text { er }\end{array}$ & $\begin{array}{l}2^{\text {nd }} \\
\text { lay } \\
\text { er }\end{array}$ \\
\hline $\begin{array}{c}\text { CA } \\
\text { 00 }\end{array}$ & $\begin{array}{c}\text { Polyes- } \\
\text { ter }\end{array}$ & $\begin{array}{l}\text { Conduc- } \\
\text { tive yarn }\end{array}$ & 150 & 114 & 32 & 16 & $0^{\circ}$ & $0^{\circ}$ \\
\hline $\begin{array}{c}\text { CA } \\
99\end{array}$ & $\begin{array}{c}\text { Polyes- } \\
\text { ter }\end{array}$ & $\begin{array}{l}\text { Conduc- } \\
\text { tive yarn }\end{array}$ & 150 & 114 & 32 & 16 & $90^{\circ}$ & $90^{\circ}$ \\
\hline $\begin{array}{c}\text { CB } \\
09\end{array}$ & $\begin{array}{c}\text { Polyes- } \\
\text { ter }\end{array}$ & $\begin{array}{l}\text { Conduc- } \\
\text { tive yarn }\end{array}$ & 150 & 114 & 32 & 16 & $0^{\circ}$ & $90^{\circ}$ \\
\hline $\begin{array}{c}\text { CB } \\
90\end{array}$ & $\begin{array}{c}\text { Polyes- } \\
\text { ter }\end{array}$ & $\begin{array}{l}\text { Conduc- } \\
\text { tive yarn }\end{array}$ & 150 & 114 & 32 & 16 & $90^{\circ}$ & $0^{\circ}$ \\
\hline
\end{tabular}

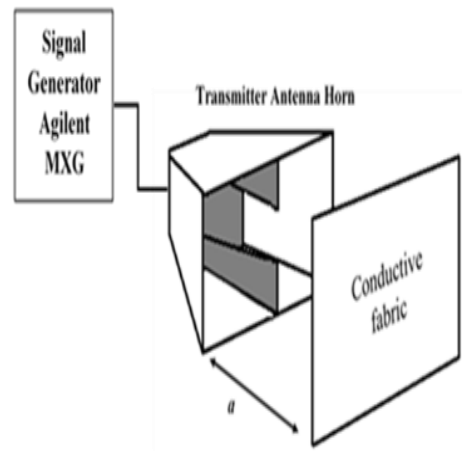

a: Distance between fabric and transmitter is changeable from $201070 \mathrm{~cm}$

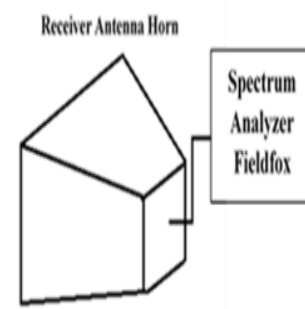




\subsection{Electromagnetic shielding effectiveness performance}

The electromagnetic shielding efficiency was evaluated according to IEEE-299 standard. In this test, the frequency used was 2000MHz. The shielded enclosure test was carried out using Signal Generator-Agilent MXG (N5183A), a spectrum analyzer Fieldfox (N9916A) and two Broadband Horn Antennas (P/N-LB8180-NF). Figure 5 shows the setup of the shielded enclosure test. The samples were tested from 1 to 2 layers with different angle arrangement as shown in Table 1. The samples were also tested on different distances of the fabric from the transmitter antenna. The distance " $a$ " in Figure 5 is denoted as the distance between fabric and transmitter. There were six different distance from the transmitter which were $20,30,40,50,60$ and $70 \mathrm{~cm}$. The fabrics were tested and placed between the two Broadband Horn Antennas. The broadband horn antennas attached with the Signal GeneratorAgilent MXG and spectrum analyzer-Fieldfox as shown in Figure 3. The Signal Generator-Agilent MXG sent the signal through the transmitter antenna and then collected by receiver antenna. The number of signals collect by the receiver antenna was measured using the spectrum analyser-Fieldfox.

\section{Result and discussion}

The results for shielded enclosure test with different distance from the transmitter antenna were in the negative (-ve) value of change $(\mathrm{dBm})$. The reason is because most of the electromagnetic waves from mobile network are not strong enough to give positive (+ve) $\mathrm{dBm}$ [26]. A small amount of $\mathrm{dBm}$ collected, shows that poor signal is received. While a higher amount $\mathrm{dBM}$ collected shows good signal being received [27]. From the study, when there is no fabric placed in between the Broadband Horn Antennas, the result of EMS was $-36.60 \mathrm{dBm}$.

\subsection{Effect of electromagnetic shielding on different distance with same angle arrangement for $0^{\circ} / 0^{\circ}$ and $90^{\circ} / 90^{\circ}$}

In this section, there are 2 layers for each sample. The first layer and the second layer have the same weft direction. Table 1 explained that sample CA00 has layered $0^{\circ}$ for the first and second layer. While, CA99 has layered $90^{\circ}$ for both, first and second layer. In Figure 6, it shows that the graph impact of electromagnetic shielding for different distance with same angle of weft direction.

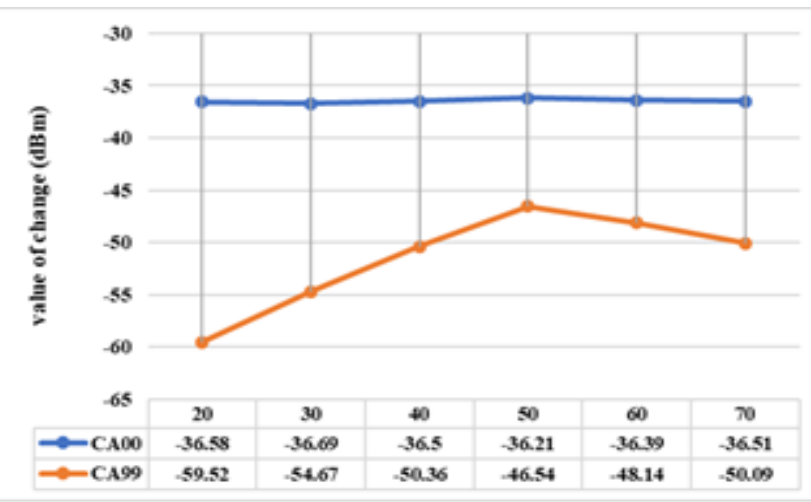

Fig 6: The effect of electromagnetic shielding with different distance but same angle of weft direction

The graph in Figure 6 shows that, sample CA90 with distance 20 $\mathrm{cm}$ from the transmitter give the lowest receiving signal, -59.52 $\mathrm{dBm}$. While, samples CA00 from Figure 6 show that the signal from all distance can still be received by the receiver antenna. The result show that the $\mathrm{dBm}$ for sample CA00 is around -36.20 to $36.70 \mathrm{dBm}$. This result indicates, that sample CA99 have better shielding capability compared to CA00.
The transmitter antenna was placed vertically and transmit the electromagnetic (EM) waves horizontally as shown in Figure 7. When the sample was placed $90^{\circ}$, the waves can be reflected. However, when the sample was placed $0^{\circ}$, the waves can go through and penetrate the shielding material. This is because, the sample and the waves were both in the same direction. Therefore, in this situation the waves can move in between the yarn in the conductive fabric.

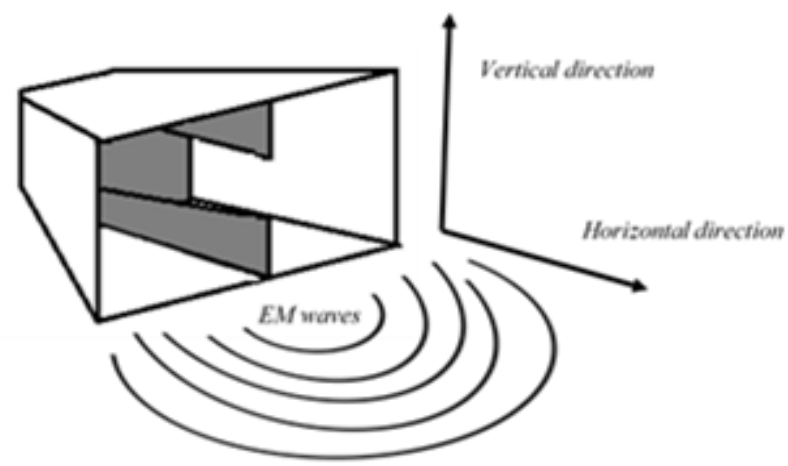

Fig 7: The electromagnetic waves and antenna direction

\subsection{Effect of electromagnetic shielding on different distance with different angle arrangement for $0 \% 90^{\circ}$ and $90 \% 0^{\circ}$}

In this section, the result of the electromagnetic shielding of different distance from the transmitter antenna and different angle are given. The first and the second layer have different weft direction $\left(0^{\circ} / 90^{\circ}\right)$ and $\left(90^{\circ} / 0^{\circ}\right)$. Sample CB09 have $0^{\circ}$ for the first layer while, the second layer is $90^{\circ}$. It is vice versa for CB90.

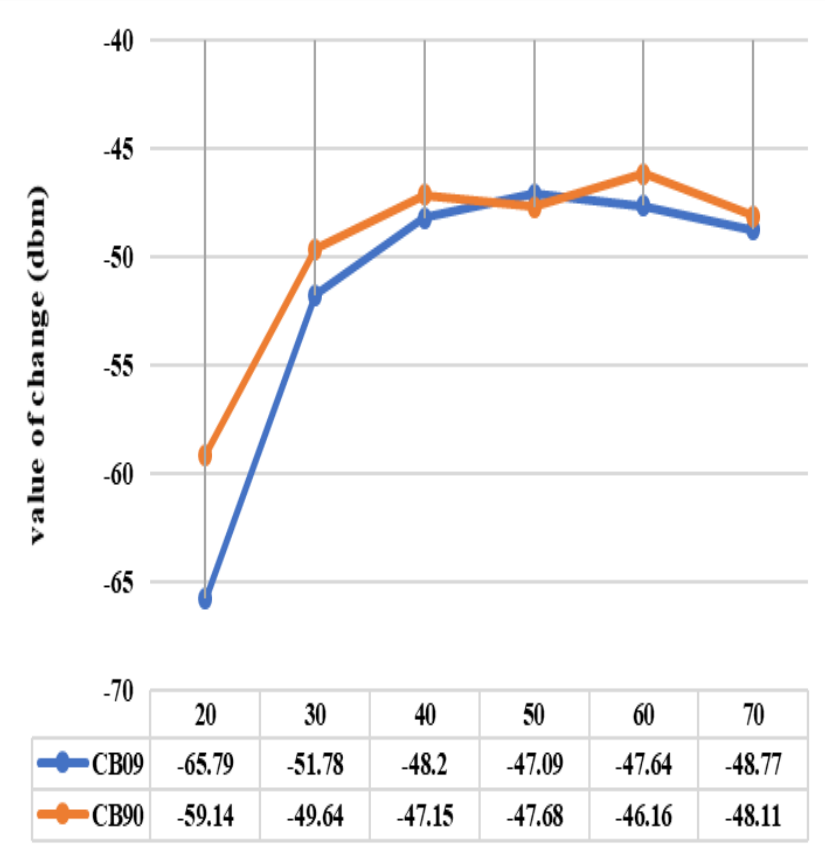

Fig 8: The effect of EM shielding with different distance and angle of weft direction

Figure 8 shows a certain relationship of EM shielding on different distance and angle of weft direction. The lower the $\mathrm{dBm}$, the better shielding properties. Sample CB90 with distance $20 \mathrm{~cm}$ can block the signal with $-59.14 \mathrm{dBm}$. However, sample CB09 with distance $20 \mathrm{~cm}$, shows better result in blocking the signal with value of change, $-65.79 \mathrm{dBm}$ compare to sample CB90.

From the results, it can be said that the first layer is the main shielding material to block or reflect the signal from the transmitter signal. For example, the result for CA99 shows that $90^{\circ}$ has 
better shielding compare to sample at $0^{\circ}$. Sample CB09 at $90^{\circ}$ as the first layer help to block the signal as shown in Figure 4. While, sample CB90 at $0^{\circ}$ as the first layer could not block most of the signal and the second layer with $90^{\circ}$ will help reflect or block the remaining signal.

\subsection{Effect of electromagnetic shielding on different distance with all different angle arrangement}

Figure 9 shows that the distance of the sample from the transmitter can give effect on receiving the signal either it can reflect the signal or not. This is because the shielding material was placed closed to the transmitter antenna, therefore when the signal is emitting, it will be immediately reflected. As well as when the shielding material was placed near to the receiver antenna, the EM waves signal will be blocked the receiver antenna. The result show that at distance $50 \mathrm{~cm}$, most of the signal becomes stronger with high value of change $(\mathrm{dBm})$ compare to $20 \mathrm{~cm}, 30 \mathrm{~cm}, 40 \mathrm{~cm}, 60 \mathrm{~cm}$ and $70 \mathrm{~cm}$ as shown in Figure 8 . The value of change at distance $50 \mathrm{~cm}$ for all samples, is around -36.60 to $-47.70 \mathrm{dBm}$. Therefore, if the sample were put far from the transmitter antenna, some of the signal will be reflected, absorbed, penetrated or spread around the room

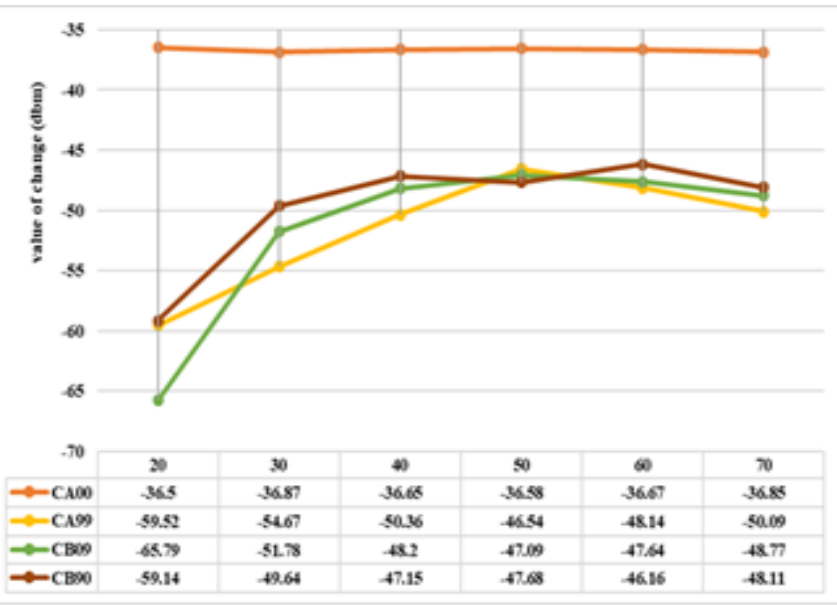

Fig 9: The effect of EM shielding on different distance

\section{Conclusion}

In the study, multi-angle layered hybrid woven fabric were produced as conductive fabric for electromagnetic shielding. The fabric was layered with different weft direction $\left(0^{\circ} / 0^{\circ}\right),\left(90^{\circ} / 90^{\circ}\right)$, $\left(0^{\circ} / 90^{\circ}\right)$ and $\left(90^{\circ} / 0^{\circ}\right)$. Each sample was tested at different distances from the transmitter antenna. This work supports that sample with different angle of weft direction give better shielding performance compared to samples with same angle of weft direction. It was found that, the distance of shielding material from the transmitter signal give effect on the reading of signal. If shielding material were placed near the transmitter antenna or receiver antenna, it will have better shielding. Sample CB09 with distance $20 \mathrm{~cm}$ from the transmitter antenna give better result compare to other samples. From the result, it shows that the different multiangle layered and distance of the conductive fabric from the transmitter gives effect on electromagnetic shielding performance.

\section{References}

[1] Duran, D. and H. Kadoğlu, Electromagnetic shielding characterization of conductive woven fabrics produced with silvercontaining yarns. Textile Research Journal, 2014. 85(10): p. 10091021.
[2] Jagatheesan, K., et al., Electromagnetic shielding behaviour of conductive filler composites and conductive fabrics-A review. 2014.

[3] Tong, S., Y.E.v. Schirnding, and T. Prapamontol, Environmental lead exposure: a public health problem of global dimensions. Bulletin of the World Health Organization, 2000. 78: p. 1068-1077.

[4] Christ, A., et al., Age-dependent tissue-specific exposure of cell phone users. Phys Med Biol, 2010. 55(7): p. 1767-83.

[5] Fernandez-Rodriguez, C.E., A.A.A. De Salles, and D.L. Davis, Dosimetric Simulations of Brain Absorption of Mobile Phone

[6] Radiation-The Relationship Between psSAR and Age. IEEE Access, 2015. 3: p. 2425-2430.

[7] Morgan, L., S. Kesari, and D. Davis, Why children absorb more microwave radiation than adults: The consequences. Journal of Microscopy and Ultrastructure, 2014. 2(4).

[8] Aydin, D., et al., Mobile phone use and brain tumors in children and adolescents: a multicenter case-control study. J Natl Cancer Inst, 2011. 103(16): p. 1264-76.

[9] Kucer, N. and T. Pamukcu, Self-reported symptoms associated with exposure to electromagnetic fields: a questionnaire study. Electromagn Biol Med, 2014. 33(1): p. 15-7.

[10] Sudan, M., Cell Phone Exposures and Headaches, Hearing Loss, and Behavioral Problems in Children. 2012: University of California, Los Angeles.

[11] Brzeziński, S., et al., Textile Multi-layer Systems for Protection Against Electromagnetic Radiation. Fibres \& Textiles in Eastern Europe, 2009(2 (73)): p. 66--71

[12] Dasa, A., et al., Effect of various parameters on electromagnetic shielding effectiveness of textile fabrics. Indian Journal of Fibre \& Textile Research, 2009. 34: p. 144-148.

[13] Maity, S., et al., Textiles in electromagnetic radiation protection Journal of Safety Engineering, 1926. 2(2): p. 11-19.

[14] Aniołczyk, H., et al., Application of electrically conductive textiles as electromagnetic shields in physiotherapy. Fibres \& Textiles in Eastern Europe, 2004(4 (48)): p. 47--50

[15] Asghar, A., et al., An alternative approach to design conductive hybrid cover yarns for efficient electromagnetic shielding fabrics. Journal of Industrial Textiles, 2017: p. 1528083717721922.

[16] The EMF Safety Shop. 2018 [cited 2018; Available from: https://www.lessemf.com/index.html.

[17] Electromagnetic shielding. 2018 [cited 2018; Available from: https://en.wikipedia.org/wiki/Electromagnetic_shielding.

[18] Perumalraj, R. and B.S. Dasaradan, Electromagnetic Shielding Effectiveness of Doubled Copper-Cotton Yarn Woven Materials. FIBRES \& TEXTILES in Eastern Europe, 2010. 18.

[19] Cheng, K.B., et al., Electromagnetic Shielding Effectiveness of the Twill Copper Woven Fabrics. Journal of Reinforced Plastics and Composites, 2016. 25(7): p. 699-709.

[20] Ortlek, H.G., T. Alpyildiz, and G. Kilic, Determination of electromagnetic shielding performance of hybrid yarn knitted fabrics with anechoic chamber method. Textile Research Journal, 2013. 83(1): p. $90-99$

[21] Das, A., et al., Effect of various parameters on electromagnetic shielding effectiveness of textile fabrics. 2009.

[22] Dordevic, Z., Textile fabric shielding electromagnetic radiation, and clothing made thereof. 1992, Google Patents.

[23] Liu, Z., X.C. Wang, and Z. Zhou, Computation of shielding effectiveness for electromagnetic shielding blended fabric. 2013

[24] Šafářová, V., M. Tunák, and J. Militký, Prediction of hybrid woven fabric electromagnetic shielding effectiveness. Textile Research Journal, 2015. 85(7): p. 673-686

[25] Stoppa, M. and A. Chiolerio, Wearable electronics and smart textiles: a critical review. Sensors, 2014. 14(7): p. 11957-11992.

[26] Polyester High Tenacity Yarn - For Industrial Application. 2017 $\begin{array}{llll}\text { [cited } 2017 \quad \text { 17]; } & \text { Available from }\end{array}$ http://www.thetexperts.com/fibers-yarns/yarns/high-tenacity/.

[27] What is meaning of negative dbm in signal strength? 20162018 [cited $2018 \quad 17 \quad$ August]; Available from https://stackoverflow.com/questions/17874852/what-is-meaning-ofnegative-dbm-in-signal-strength/31869981\#.

[28] Moyers, E. Why is almost everything negative in Wireless? 2015 Available from: https://community.cisco.com/t5/small-businesssupport-documents/why-is-almost-everything-negative-inwireless/ta-p/3159743 